\title{
A Full-Plane Block Kalman Filter for Image Restoration
}

\author{
Stuart Citrin, Member, IEEE, and Mahmood R. Azimi-Sadjadi, Senior Member, IEEE
}

\begin{abstract}
A new two-dimensional (2-D) block Kalman filtering method is presented which uses a full-plane image model to generate a more accurate filtered estimate of an image that has been corrupted by additive noise and full-plane blur. Causality is maintained within the filtering process by employing multiple concurrent block estimators. In addition, true state dynamics are preserved, resulting in an accurate Kalman gain matrix. Simulation results on a test image corupted by additive white Gaussian noise are presented for various image models and compared to those of the previous block Kalman filtering methods.
\end{abstract}

\section{INTRODUCTION}

$\mathrm{K}$ ALMAN filtering has been known to be an effective method for signal processing and control applications [1], [2]. The extension of the standard one-dimensional (1-D) Kalman filtering to the two-dimensional (2-D) case such as in image processing, however, creates various problems. To maintain the proper state dynamics within both the state and the error covariance equations [1], [2] and to design an optimal Kalman filter, a large state vector and correspondingly large error covariance matrices would be involved. This, obviously, leads to an excessively large amount of storage and computations. A number of researchers introduced various filtering schemes [3]-[6] to overcome these problems. The idea of the reduced update Kalman filtering (RUKF) [4]-[6] is to partition the state vector into two segments-the "local state" and the "global state." The "local state" propagates in both dimensions during the filtering process and consists of a group of pixels, in the region of support (ROS) of the model, which are spatially close to the points being estimated. The "global state," however, contains those previously estimated pixels needed to estimate the future ones. Substantial computational saving is achieved by using only the local state in the filtering process and by transferring the data from the global state into the local state whenever the initial estimate is generated. Pixels which are not included in the local state tend to be less correlated and provide little information for generating the initial estimate due to the Markovian assumption.

The ROS of the 2-D model can have various geometry and types, namely, causal, semi-causal (or half-plane),

Manuscript received May 14, 1990; revised February 17, 1992.

The authors are with the Department of Electrical Engineering, Colorado State University, Fort Collins, CO 80523

IEEE Log Number 9202153 and noncausal (or full-plane) [7]. Although noncausal models are shown [7] to provide better match to the actual image correlations, causal support was widely used in almost all of the previous methods. However, when a causal model is used more than half of the pixels in the adjoining support are ignored in generating the initial estimate. Therefore, it is desirable to devise a way to generate a full-plane model for more accurate estimation while maintaining the causality within the filtering process.

In this paper a full-plane block Kalman filtering scheme is developed which uses multiple concurrent block estimators to maintain causality within the actual filtering process. By using this method true state dynamics are preserved without having to store or make calculations on excessively large error covariance matrices. By using a full-plane model, more accurate modeling of the image process can be achieved, which leads to a more accurate Kalman gain matrix, and hence, improvements in the signal-to-noise ratio (SNR) in the filtered images.

\section{Propagation of the State in the Block State-Space Model}

Consider an image of size $M \times M$ which is scanned vectorially from left to right and top to bottom in block rows of width $N_{\mathrm{l}}$. The image is assumed to be represented by a zero-mean vector Markov process. Each block within a block row is of size $N=N_{1} \times N_{2}$, where $N_{1}$ is the number of rows of pixels within a block; $N_{2}$ is the number of columns in a block, and $N$ is the number of pixels in each block. A block row is defined as a strip of blocks extending across the image from left to right and is of size $N_{1} \times M$. A block row contains $M / N_{2}$ blocks, assuming that $M$ is divisible by $N_{2}$. The process is illustrated in Fig. 1. The pixels within a block are arranged in row-ordered form. A processing strip, hereafter called a strip, consists of three block rows. The goal is to estimate the blocks in the middle block row. The upper and lower block rows consist of block estimates generated to provide support for the blocks in the middle block row. Two estimates of the blocks in the middle block row need to be generated. The first estimates are generated solely to provide support to the second estimator. The second block estimates in the middle block row will be saved as the final filtered estimate.

The local state-space model for the image process is 
given by

$$
\left[\begin{array}{c}
X_{0}(k) \\
X_{1}(k) \\
\vdots \\
X_{8}(k)
\end{array}\right]=A\left[\begin{array}{c}
X_{0}(k-1) \\
X_{1}(k-1) \\
\vdots \\
X_{8}(k-1)
\end{array}\right]+\left[\begin{array}{c}
U_{0}(k) \\
U_{1}(k) \\
\vdots \\
U_{8}(k)
\end{array}\right]
$$

or

$$
\boldsymbol{X}(k)=A X(k-1)+B U(k)
$$

where $\boldsymbol{X}(k)$ is the current state vector consisting of 9 blocks $X_{i}(k), i \in[0,8] ; \boldsymbol{X}(k-1)$ is the past state vector consisting of 9 blocks $X_{i}(k-1) i \in[0,8]$, and $U(k)$ is a zero mean white driving noise vector process and is of size $(9 \times N) \times 1$. The first $5 \times N$ elements of $U(k)$ are equal to zero.

The spatial positions of $X_{i}(k)$ at a given iteration " $k$ "' are shown in Fig. 1. The peculiar numbering of these blocks is solely chosen to provide easier programming by getting all the blocks which are to be estimated, i.e., blocks $5,6,7$, and 8 , numerically close together and their support numerically adjacent. The blocks which are not filtered estimates are obtained by shifting the blocks within the state as the state advances to the right, so that the previously estimated blocks occupy the proper spatial positions within the state. Fig. 2 illustrates the state propagation along horizontal direction with each iteration.

The supports for blocks $5,6,7$, and 8 are given below:

\begin{tabular}{cc}
\hline Filtered Block & Support \\
\hline$X_{5}(k)$ & $X_{i}(k-1), i=4,5,6,7$ \\
$X_{6}(k)$ & $X_{i}(k-1), i=6$ \\
$X_{7}(k)$ & $X_{i}(k-1), i=4,5,6,7$ \\
$X_{8}(k)$ & $X_{i}(k-1), i=0,1,2,3,4,5,6,7,8$ \\
\hline
\end{tabular}

This results in an $A$ matrix of size $(9 \times N) \times(9 \times N)$ which is given by

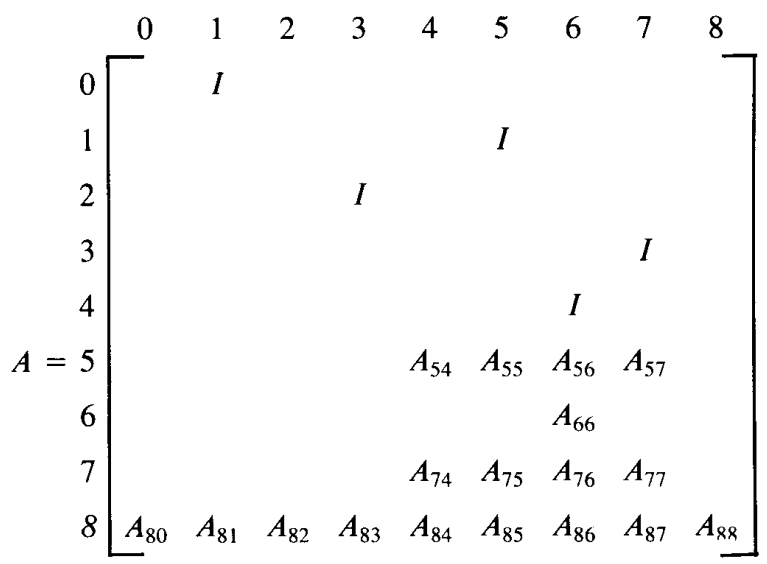

where all submatrices of $A$ not filled in above are zero matrices. Each submatrix of $A$ is of size $N \times N$.

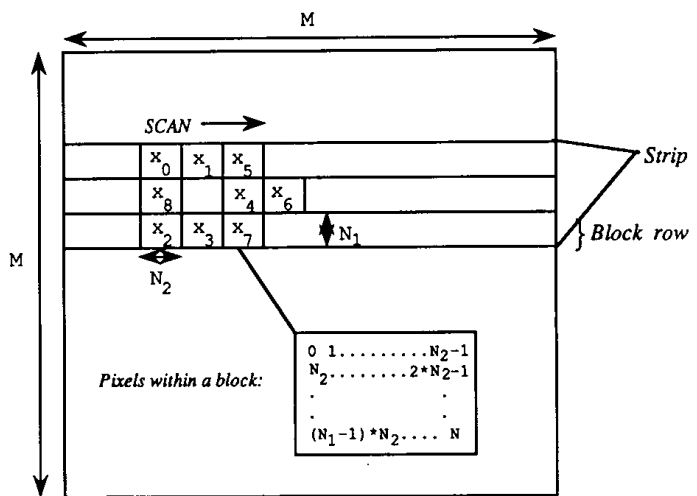

Fig, 1. Size of blocks, block row, state, and numbering of pixels within a block.

\begin{tabular}{|l|l|l|l|l}
\hline$x_{0}$ & $x_{1}$ & $x_{5}$ & -7 & 1 \\
\hline$x_{8}$ & & $x_{4}$ & $x_{6}$ & -1 \\
\hline$x_{2}$ & $x_{3}$ & $x_{7}$ & & -1 \\
\hline
\end{tabular}

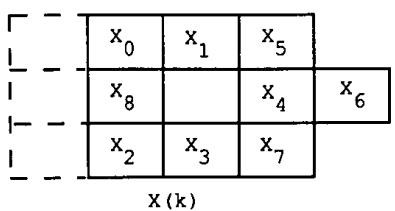

Fig. 2. Propagation of the state. $X_{0}(k)$ and $X_{1}(k-1)$ occupy the same spatial position.

The procedure in each strip begins by advancing the strip one block row down. The propagation of the state along the boundaries will be discussed later. The state propagates along the strip from left to right and as it advances blocks $0,1,2,3$, and 4 are shifted from the previous state and blocks $5,6,7$, and 8 are estimated using four concurrent estimators. Block 5 is re-estimated to avoid the storage of this block from the previous block row, which could have resulted in large error covariance matrices. Block 7 is an intermediate estimate of data that will again be estimated as blocks 6 and 8 when the strip is advanced to the next block row. Block 6 , which is ahead of block 8 , is estimated based upon the past block 6 in the same block row to provide the right side support of block 8. The filtered estimate of block 8 with a full-plane of support is the only block estimate which is saved.

As can be seen, by using these multipole concurrent block estimators causality is maintained within the actual filtering process. Furthermore, re-estimating those blocks along the upper and lower block rows avoids the need to store large error covariances associated with these states, thus resulting in substantial reduction in computation efforts and storage requirements. The only disadvantage is that the recursion occurs in one direction. 
III. Parameter Estimation for the Image Model

In this section a procedure is given to obtain the model parameters for the four estimators $\left(X_{i}(k), i=5,6,7,8\right)$ described in Section II. The model parameters to be estimated are the $A_{i}$, i.e., the $i$ th block row of the $A$ matrix and the correlation matrices $Q_{U i j} \triangleq E\left[U_{i}(k) U_{j}^{T}(k)\right], i, j=$ $0,1, \cdots 8$, where $E[\cdot]$ is the expectation operator. These are the diagonal submatrices of the covariance matrix, $Q_{U}$, of the driving process, $U(k)$.

Let us begin by considering the first block estimator $X_{6}(k)$ which has only one support block. To obtain the model parameters $A_{6}$ and $Q_{U / 6}$ for this block estimator we extract the corresponding row of (1) i.e.:

$$
X_{6}(k)=A_{6} X(k-1)+U_{6}(k) .
$$

Ignoring the zero portion of $A_{6}$, we simply obtain

$$
X_{6}(k)=A_{66} X_{6}(k-1)+U_{6}(k)
$$

where $X_{6}(k)$ and $U_{6}(k)$ are of size $N \times 1$ and $A_{66}$, which is the seventh submatrix of $A_{6}$, is of size $N \times N$. By postmultiplying by $X_{6}^{T}(k-l)$, taking the expectation, and using the orthogonality principle [1]:

$$
E\left[U_{6}(k) X_{6}^{T}(k-1)\right]=0
$$

yields

$$
\rho_{66}(l)=A_{66} \rho_{66}(l-1)+Q_{U 66} \delta(l)
$$

where

$$
\rho_{i j}(l) \triangleq E\left[X_{i}(n) X_{j}(n-l)\right] .
$$

Transposing (5a) and using the property $\rho_{i j}^{T}(l)=\rho_{j i}(-l)$ gives

$$
\rho_{66}(-l)=\rho_{66}(1-l) A_{66}^{T}+Q_{U 66} \delta(l)
$$

which is the normal equation for this estimator. Plugging $l=0,1$ in this equation gives the following vector YuleWalker equation

$$
\left[\begin{array}{ll}
\rho_{66}(0) & \rho_{66}(1) \\
\rho_{66}^{T}(1) & \rho_{66}(0)
\end{array}\right]\left[\begin{array}{l}
I \\
-A_{66}^{T}
\end{array}\right]=\left[\begin{array}{l}
Q_{U 66} \\
0
\end{array}\right]
$$

which can be rearranged and solved to give $Q_{U 66}$ and $A_{66}$.

To obtain the parameters $A_{5}$ and $Q_{U 55}$ for the second block estimator $X_{5}(k)$, and $A_{7}$ and $Q_{U 77}$ for the third estimator $X_{7}(k)$, the same procedure can be repeated. For example for the second estimator the state equation is

$$
X_{5}(k)=A_{5} X(k-1)+U_{5}(k)
$$

which is obtained by extracting the sixth block row of (1). Alternatively, we have

$$
X_{5}(k)=\left[\begin{array}{llll}
A_{54} & A_{55} & A_{56} & A_{57}
\end{array}\right]\left[\begin{array}{c}
X_{4}(k-1) \\
X_{5}(k-1) \\
X_{6}(k-1) \\
X_{7}(k-1)
\end{array}\right]+U_{5}(k) .
$$

Using a similar procedure and invoking the orthogonality principle gives the relevant vector Yule-Walker equation as

$$
\begin{gathered}
{\left[\begin{array}{ccccc}
\rho_{55}(0) & \rho_{54}(1) & \rho_{55}(1) & \rho_{56}(1) & \rho_{57}(1) \\
\rho_{54}^{T}(1) & \rho_{44}(0) & \rho_{45}(0) & \rho_{46}(0) & \rho_{47}(0) \\
\rho_{55}^{T}(1) & \rho_{54}(0) & \rho_{55}(0) & \rho_{56}(0) & \rho_{57}(0) \\
\rho_{56}^{T}(1) & \rho_{64}(0) & \rho_{65}(0) & \rho_{66}(0) & \rho_{67}(0) \\
\rho_{57}^{T}(1) & \rho_{74}(0) & \rho_{75}(0) & \rho_{76}(0) & \rho_{77}(0)
\end{array}\right]} \\
\\
{\left[\begin{array}{c}
\mathrm{I} \\
-\mathrm{A}_{54}^{T} \\
-\mathrm{A}_{55}^{T} \\
-\mathrm{A}_{56}^{T} \\
-\mathrm{A}_{57}^{T}
\end{array}\right]=\left[\begin{array}{c}
\mathrm{Q}_{\mathrm{U} 5} \\
0 \\
0 \\
0 \\
0
\end{array}\right]}
\end{gathered}
$$

The solution of this system of equation provides $Q_{\mathrm{U} 55}$ and $A_{5 i}, i=4,5,6,7$. For the third estimator, $X_{7}(k)$, we simply replace $X_{5}(k)$ with $X_{7}(k), U_{5}(k)$ with $U_{7}(k), A_{5 i}$ with $A_{7 i}$ and $\rho_{5 i}$ with $\rho_{7 i}(i=4,5,6,7)$ in (9) and (10).

For the estimation of the model parameters for the last block estimator, $X_{8}(k)$, we have

$$
\begin{gathered}
{\left[\begin{array}{ccccc}
\rho_{88}(0) & \rho_{80}(1) & \rho_{81}(1) & \cdots & \rho_{88}(1) \\
\rho_{80}^{T}(1) & \rho_{00}(0) & \rho_{01}(0) & \cdots & \rho_{08}(0) \\
\rho_{81}^{T}(1) & \rho_{10}(0) & \rho_{11}(0) & \cdots & \rho_{18}(0) \\
\vdots & \vdots & \vdots & & \vdots \\
\rho_{88}^{T}(1) & \rho_{80}(0) & \cdots & \cdots & \rho_{88}(0)
\end{array}\right]\left[\begin{array}{c}
I \\
-A_{80}^{T} \\
-A_{81}^{T} \\
\vdots \\
-A_{88}^{T}
\end{array}\right]} \\
\quad\left[\begin{array}{c}
Q_{U 88} \\
0 \\
0 \\
\vdots \\
0
\end{array}\right]
\end{gathered}
$$

which can be solved to give $Q_{U 88}$ and $A_{8 i}, i \in[0,8]$. It should be noted that not all the correlation matrices in the above vector Yule-Walker equations need to be calculated due to the spatial equivalencies such as

$$
\begin{aligned}
\rho_{i i}(0) & =\rho_{j j}(0), \quad i \neq j \\
\rho_{i j}(-n) & =\rho_{j i}^{T}(-n), \quad n \text { any integer }
\end{aligned}
$$

and

$$
\rho_{45}(0) \approx \rho_{74}(0) \text {. }
$$

By using these and similar equivalencies, considerable computation time can be saved.

So far we have obtained all the required submatrices of matrix $A$ and also identified the diagonal submatrices of $Q_{U}, Q_{U i}, i=j$. In order to complete the modeling process 
the off-diagonal submatrices $Q_{U i j}, i \neq j$, have to be determined. For the $Q_{U 56}$ matrix, post-multiply both sides of $(9)$ by $X_{6}^{T}(k)$ and use the orthogonality principle, $E\left[U_{5}(k) X_{6}^{T}(k-1)\right]=0$ which gives

$$
Q_{U 56}=\rho_{56}(0)-\left[\begin{array}{llll}
A_{54} & A_{55} & A_{56} & A_{57}
\end{array}\right]\left[\begin{array}{c}
\rho_{46}(-1) \\
\rho_{56}(-1) \\
\rho_{66}(-1) \\
\rho_{76}(-1)
\end{array}\right] \text {. }
$$

Similarly for $Q_{U 76}$ and $Q_{U 57}$. For $Q_{U 58}$ we obtain

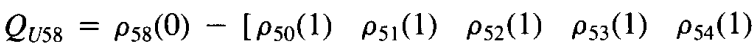

$$
\begin{aligned}
& \text { - } \left.\rho_{55}(1) \quad \rho_{56}(1) \quad \rho_{57}(1) \quad \rho_{58}(1)\right] A_{8}^{T} \text {. }
\end{aligned}
$$

similarly, for $Q_{U 78}$ and $Q_{U 68}$. The remaining off-diagonal submatrices can be obtained by the realtionship, $Q_{U j i}=$ $Q_{U i j}^{T}$. For $i \in[0,4], j \in[0,8]$, and $i \in[5,8], j \in[0,4]$ $Q_{U i j}=0$, i.e., a zero submatrix as the corresponding blocks are obtained solely by the shifting process.

\section{Kalman Filtering Process}

Once all the model parameters are identified, the block dynamic (1) can be formed. The observation equation in block form is

$$
Z(k)=H \boldsymbol{X}(k)+V(k)
$$

where $Z(k)$ is the corrupted image or observation vector of size $(4 \times N) \times 1, V(k)$ is the observation noise vector of the same size as $Z(k)$ containing a scalar zero mean white Gaussian additive noise $v(k)$ with variance $\sigma_{v}^{2}$, and $H$ is a $(4 \times N) \times(9 \times N)$ matrix containing the elements of the point spread function (PSF) of the noncausal blur. For the case of an image corrupted solely by additive noise $H$ becomes

$$
H=\left[\begin{array}{lllllllll}
0 & 0 & 0 & 0 & 0 & I & 0 & 0 & 0 \\
0 & 0 & 0 & 0 & 0 & 0 & I & 0 & 0 \\
0 & 0 & 0 & 0 & 0 & 0 & 0 & I & 0 \\
0 & 0 & 0 & 0 & 0 & 0 & 0 & 0 & I
\end{array}\right]
$$

where 0 represents a zero submatrix each of size $N \times N$. The Kalman filter equations for the system in (1) and (14) are [1], [2]

$$
\begin{aligned}
P_{b}(k) & =A P_{a}(k-1) A^{T}+B Q_{U} B^{T} \\
K(k) & =P_{b}(k) H^{T}\left(H P_{b}(k) H^{T}+Q_{V}\right)^{-1} \\
\hat{\boldsymbol{X}}(k) & =A \hat{\boldsymbol{X}}(k-1) \\
\hat{\hat{X}}(k) & =\hat{\boldsymbol{X}}(k)+K(k)[Z(k)-H \hat{\boldsymbol{X}}(k)] \\
P_{a}(k) & =[I-K(k) H] P_{b}(k)
\end{aligned}
$$

where $\hat{\boldsymbol{X}}(k)$ is the a priori (before updating) estimate, $\hat{\hat{\boldsymbol{X}}}(k)$ is the a posteriori (after updating) estimate; $P_{b}(k)$ is the $a$ priori error covariance matrix defined by

$$
P_{b}(k) \triangleq E\left[(\boldsymbol{X}(k)-\hat{\boldsymbol{X}}(k))(\boldsymbol{X}(k)-\hat{\boldsymbol{X}}(k))^{T}\right]
$$

$P_{a}(k)$ is the a posteriori error covariance matrix defined by

$$
P_{a}(k) \triangleq E\left[(\boldsymbol{X}(k)-\hat{\hat{X}}(k))(\boldsymbol{X}(k)-\hat{\hat{X}}(k))^{T}\right]
$$

$K(k)$ is the Kalman gain matrix and $Q_{U}$ and $Q_{V}$ are correlation matrices of the independent processes $U$ and $V$. The state is reinitialized at the beginning of each strip. With the exception of the boundaries of the image, only the estimate $\hat{\boldsymbol{X}}_{8}(k)$ is saved as a final estimate. After a strip is processed, one advances a block row and starts a new strip without using any filtered estimates from the prior strip. Thus recursion only occurs along a strip.

\section{A. Boundary Conditions}

There are four boundary conditions to be considered, which correspond to top, bottom, left, and right edges of the image. The condition at the beginning of a strip (left boundary) is related to the state at iteration $k=-1$. The blocks that are outside the image, i.e., $X_{i}(-1), i=0,2$, 8 are initialized to the mean of the image while those inside the image, i.e., $X_{i}(-1), i=1,3,4,5,6,7$, are initialized to the observed noisy data corresponding to those in spatial position.

At the beginning of each strip one also needs to initialize the $P_{a}(-1)$ matrix. For those estimates spatially located outside the image, the diagonal elements of $P_{a}(-1)$ are chosen as the variance of the image since the mean is used as the estimate. For those estimates using the observation, the diagonal elements are the variance of the noise, since this corresponds to the squared error associated with these estimates. The off-diagonal elements of $P_{a}(-1)$ are expected to be zero.

Next, we consider the boundary conditions at the right edge of the image, i.e., at the end of a strip. As the state approaches the right side boundary of the image at iteration $k=\left(M / N_{2}-1\right)-3$ the image is processed like the previous iterations, with block $X_{8}(k)$ being saved as a final filtered estimate. For the three subsequent and final iterations in the strip, both $X_{6}(k)$ and $X_{8}(k)$ are saved as final estimates for their proper spatial positions. This ensures that all spatial positions of the final filtered image contain filtered data without having to contend with the fact that no observation exists outside the right boundary of the image.

For the first strip of the image located spatially within the image, estimate $X_{5}(k)$ is saved as the final filtered estimate for the first block row and estimate $X_{8}(k)$ is saved as a final filtered estimate for the second block row. The first two blocks, and also the last block of the first block row are left unfiltered, i.e., the observation is used as the final estimate. The last block row is processed the same as the first block row except that $X_{7}(k)$ is saved as the final 
filtered estimate. The first two and last blocks of this block row are also left unfiltered.

\section{IMPLEMENTATION AND RESULtS}

In this section the effectiveness of the proposed fullplane Kalman filter is examined on the "Lena" image in Fig. 3. The corrupted image was obtained by adding a white Gaussian noise to the original image to achieve a SNR of $5 \mathrm{~dB}$. The resultant corrupted image is shown in Fig. 4.

For a given pixel in the block, the diagonal elements of matrix $Q_{U}$ represent the corresponding average squared error in the modeling process. The smaller a given element is, the better the fit of the model for the corresponding pixel. These values are listed in Table I for pixels in blocks $5,6,7$, and 8 . Comparison of these values indicates that the smallest values occur for the pixels of submatrix $Q_{U 88}$ which correspond to the block with the fullplane of support, i.e., $X_{8}(k)$. A better fitted model obviously results in lower values of the Kalman gain, which in turn result in decreased dependency of the estimate on the noisy observation as can be observed by inspection of (16a)-(16e). As a result, an improved SNR can be expected for block $\hat{\hat{X}}_{8}(k)$ compared to the blocks with a nonsymmetric half plane (NSHP) plane or single block of support.

The diagonal elements of the converged $P_{a}$ matrix for a given block which represent the predicted squared of the estimation error indicate the performance of a particular estimator. The diagonal elements of the $P_{a}$ matrix corresponding to blocks $5,6,7$, and 8 (i.e., the lower 16 diagonal elements) are also listed in Table I. Within each block, the values are the lowest corresponding to those pixels with the closer and most extensive support. Between blocks, the values are lower as the plane of support gets larger; that is, these diagonal values are the highest for the block 6 estimator and the lowest for the block 8 estimator. This indicates once again that the full-plane block estimator provides a better filtering and estimation than those of the symmetric and the NSHP filters.

Equations (16a), (16b), and (16e) were executed in a program to generate the Kalman gain matrix. $P_{a}(k)$ and $K(k)$ matrices were found to converge to steady state within 25 iterations. The magnitude of the steady-state values, especially the diagonal elements of the submatrices corresponding to the observation blocks, i.e, submatrices $55,66,77$, and 88 represent a measure of the goodness of fit of the model and of the actual filtering process. The diagonal elements are found to be the lowest for the $K_{88}$ submatrix which again suggests closer modeling for the block with a full-plane of support. This consequently results in a higher SNR.

The consistency test is also performed to demonstrate the adequancy of the model by determining the convergence behavior of the innovation sequence and showing that it is asymptotically zero-mean white Gaussian. The covariance matrix and the mean vector for this sequence

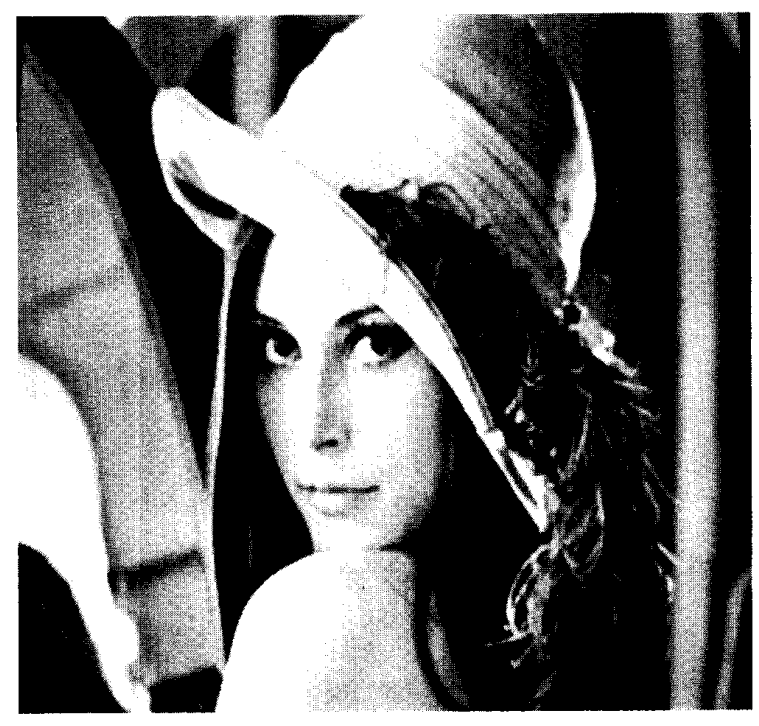

Fig. 3. Original Lena image.

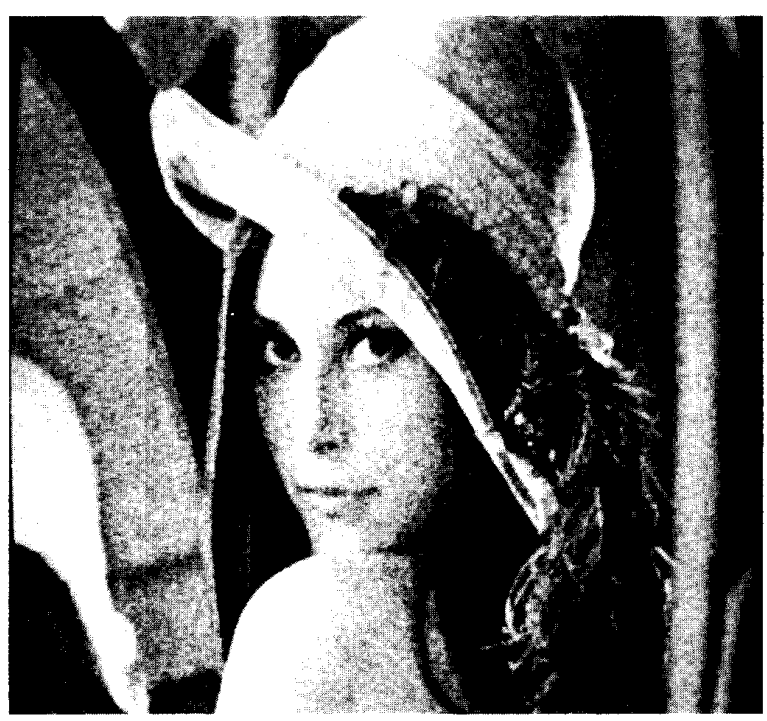

Fig. 4. Lena image corrupted by $5-\mathrm{dB}$ noise.

were computed for all the estimators over the entire image. The results reveal that the estimator with full-plane support, the results of which are saved as final estimates, satisfies the consistency test.

Figs. 5 and 6 show the images filtered using a 2 by 2 and 1 by 1 block sizes, respectively. Table II summarizes the statistical data and results pertaining to these images. As can be observed from this table, the means of all the images are approximately equal, indicating that the estimators are unbiased. The variances of the filtered images are lower than the original image, implying some loss of information in these images which can be largely attrib- 
TABLE I

Diagonal Values of $Q_{U}$ AND $P_{a}$

\begin{tabular}{lccc}
\hline Block & Pixel & Diagonal Values of $Q_{U}$ & Diagonal Values of $P_{a}$ \\
\hline 5 & 1 & 66.4 & 117.0 \\
5 & 2 & 127.2 & 134.7 \\
5 & 3 & 34.0 & 91.1 \\
5 & 4 & 52.3 & 104.0 \\
6 & 1 & 104.6 & 115.0 \\
6 & 2 & 291.9 & 168.3 \\
6 & 3 & 113.8 & 115.0 \\
6 & 4 & 316.9 & 177.4 \\
7 & 1 & 31.6 & 90.3 \\
7 & 2 & 56.4 & 102.0 \\
7 & 3 & 70.0 & 118.9 \\
7 & 4 & 149.1 & 143.1 \\
8 & 1 & 26.3 & 71.3 \\
8 & 2 & 27.0 & 72.3 \\
8 & 3 & 27.0 & 71.5 \\
8 & 4 & 26.4 & 73.3 \\
\hline
\end{tabular}

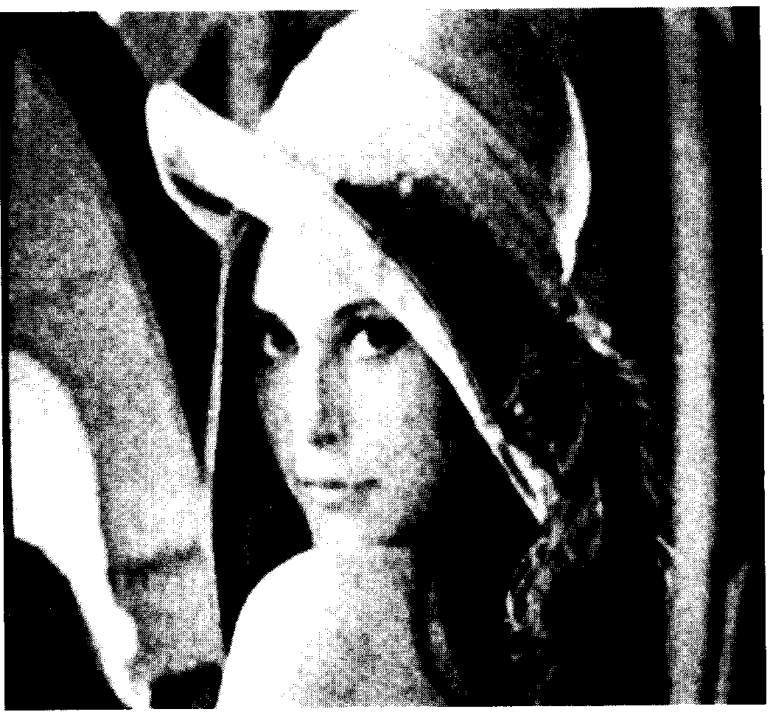

Fig. 5 . Filtered image using 2 by 2 block size.

uted to oversmoothing of the edges. A higher SNR is obtained with the larger block size ( 2 by 2 ). The processing times shown in Table II include compile time. Execution was done on a sequential machine, namely a MicroVAX 3600 .

The diagonal elements of matrix $Q_{U}$ are smaller when a 1 by 1 block size is used, indicating more accurate modeling than the 2 by 2 block size case. This was expected to occur since with a 1 by 1 block size there are more adjacent support pixels. In spite of this closer modeling, the 2 by 2 block size resulted in a filtered image with a higher SNR. The better SNR performance for the latter case is mainly attributed to the use of a higher order Marovian with greater averaging out of the noise.

Visual evaluation of the images in Figs. 4-6 shows that, subjectively, the filter is effective at reducing the amount

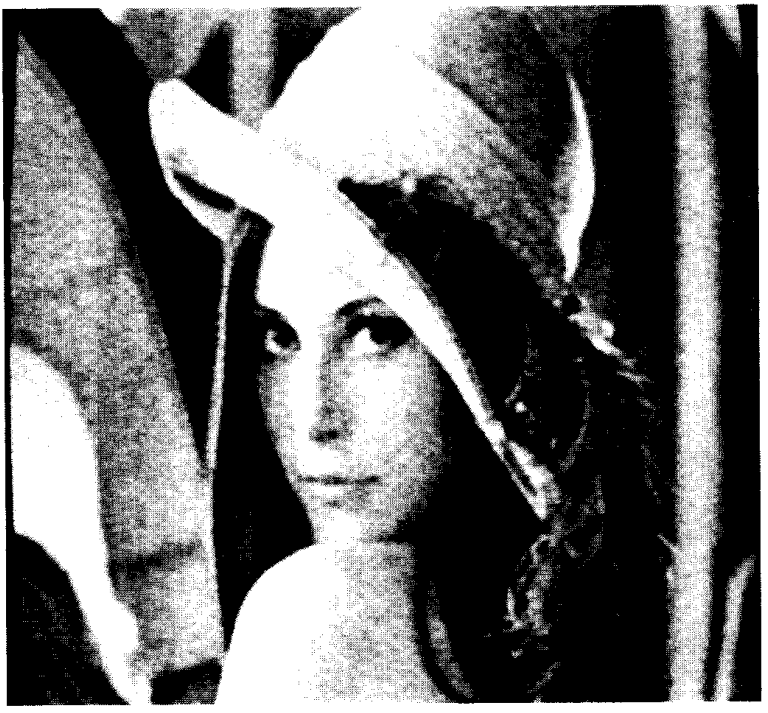

Fig. 6. Filtered image using 1 by 1 block size.

of the noise present while causing minimal blurring of the edges.

To compare the results with those of the other methods, the image was filtered using the scalar reduced update Kalman filter (RUKF) [3] and the block diagonal nonsymmetric half-plane filter (BDNSHP) [5] (see Figs. 7 and 8). For the RUKF the size of the NSHP support was 2 by 2 and the resulting SNR was $10.2 \mathrm{~dB}$. The case of 1 by 1 block size in this paper, which would have a support of approximately 3 by 3 , resulted in a filtered image with a SNR of $13.2 \mathrm{~dB}$.

Using the BDNSHP filter [5] with a block size of 3 by 6 and a support for each pixel of 12 pixels the SNR was measured to be $8.74 \mathrm{~dB}$. This is to be compared with the 1 by 1 case in this paper, where each pixel had 8 pixels of support and the SNR obtained was $13.2 \mathrm{~dB}$. 
TABLE II

Statistical Summary of Results

\begin{tabular}{lcccc}
\hline & Original & Corrupted & Filtered 2 by 2 & Filtered 1 by 1 \\
\hline Mean & 123.6 & 124.1 & 123.6 & 123.6 \\
Variance & 2298 & 2967 & 2230 & 2186 \\
Mean Error Variance & 0 & 69.2 & 87.3 & 100.5 \\
Block 8 average & - & - & 72.2 & 82.8 \\
$\quad$ diagonal $P_{a}$ Value & $\infty$ & 5.17 & 13.9 & 13.2 \\
SNR (dB) & - & - & 70.0 & 43.3 \\
Average diagonal $Q_{U}$ & - & - & 206.8 & 129.3 \\
$\quad$ Block 55 & - & - & 76.8 & 46.3 \\
$\quad$ Block 66 & - & - & 26.7 & 18.3 \\
$\quad$ Block 77 & - & & $16.2 \mathrm{~min}$ & $8.7 \mathrm{~min}$. \\
$\quad$ Block 88 & & & & \\
Processing Time & & & & \\
\hline
\end{tabular}

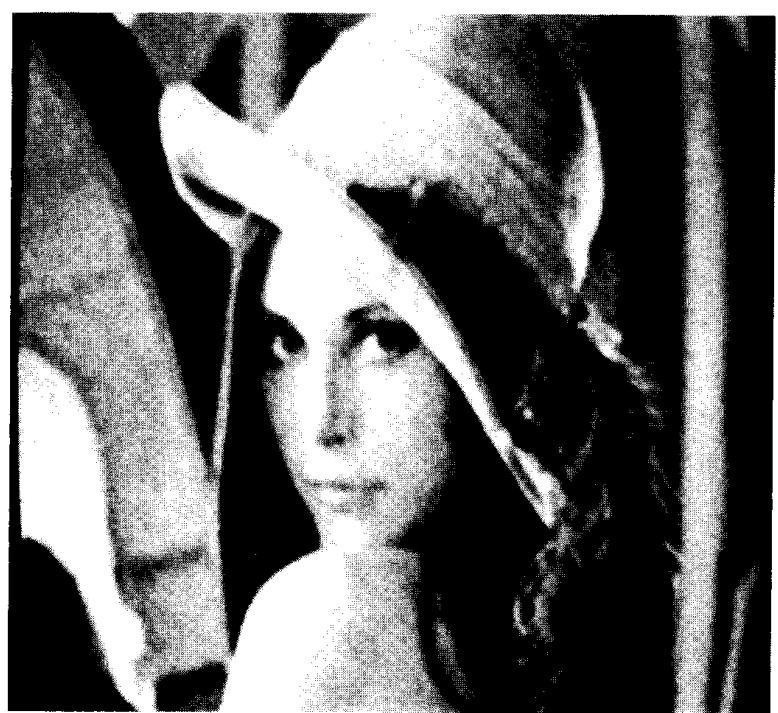

Fig. 7. Filtered image using scalar RUKF method.

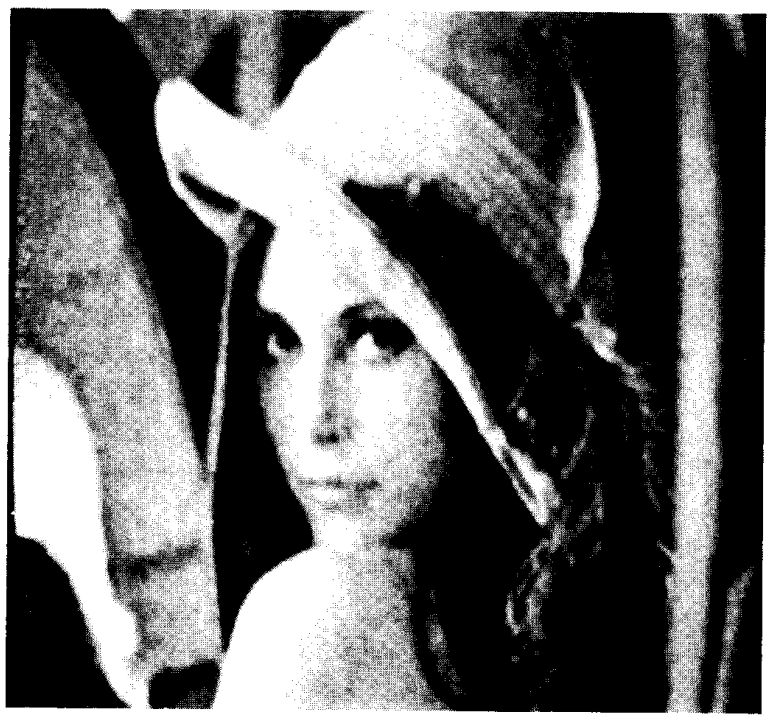

Fig. 8. Filtered image using BDNSHP method.

\section{CONClusion}

A method of block Kalman filtering of digital images using four concurrent block estimators was developed. This method uses an image model with full-plane of support and yet maintains causality within the actual filtering process. Furthermore, true state dynamics are preserved without requiring excessively large amounts of storage for the state and the error covariance matrices. The proposed scheme results in closer modeling of the image process when compared with the single estimator and the three estimator (quarter plane of support) cases in [8]. The comparison with other scalar and block Kalman filtering methods indicates considerable improvement in SNR. The final filtered images showed an approximately $9-\mathrm{dB}$ improvement in SNR with minimal loss of edge information. The proposed modeling is well suited for the situations when the PSF of the blur occurs in all directions [10].

\section{REFERENCES}

[1] A. P. Sage and J. L. Melsa, Estimation Theory with Applications to Communications and Control. New York: McGraw-Hill, 1971.

[2] "Special issue on application of Kalman filtering," IEEE Trans. Autom. Contr., vol. AC-28, Mar. 1983.

[3] J. W. Woods and V. K. Ingle, "Kalman filtering in two-dimensions Further results," IEEE Trans. Acoust., Speech Signal Processing, vol. ASSP-29, pp. 188-197, Apr. 1981.

[4] B. R. Suresh and B. A. Shenoi, "New results in two-dimensional Kalman filtering with applications to image restorations," IEEE Trans. Circuits Syst., vol. CAS-28, pp. 307-319, April 1981.

[5] M. R. Azimi-Sadjadi, S. Bannour, and S. Citrin, "A 2-D adaptive diagonal block Kalman filter for nonsymmetric half plane image models," in Proc. IEEE Int. Symp. on Circuits and Systems, Portland, OR, May 1989, pp. 1528-1531.

[6] M. R. Azimi-Sadjadi and S. Bannour, "Two dimensional adaptive block Kalman filtering of SAR imagery,"' IEEE Trans. Geosci. Remote Sensing, vol. 29, pp. 742-753, Sept. 1991.

[7] S. Ranganath and A. K. Jain, "Two-dimensional linear prediction Models-Part I: spectral factorization and realization,"' IEEE Trans. Acoust., Speech Signal Processing, vol. ASSP-33, pp. 280-299, February 1985 .

[8] S. Citrin, "Two-dimensional block Kalman filtering using multiple estimators," M.S. thesis, Colorado State Univ., 1990.

[9] S. Citrin and M. R. Azimi-Sadjadi, "A full-plane block Kalman filter for image estimation," in Proc. ISCAS'90, New Orleans, LA, May 1990 , pp. $767-770$

[10] A. M. Tekalp, H. Kaufman, and J. W. Woods, "Identification of image and blur parameters for the restoration of noncausal blur," IEEE Trans. Acoust. Speech Signal Processing, vol. ASSP-34, pp. 963972, Aug. 1986. 


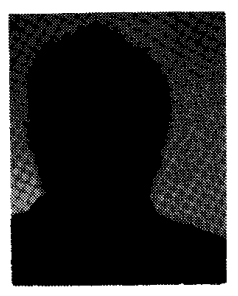

Stuart Citrin (S'89-M'90) was born in New York City in 1953. He had received the B.A. degree in psychology from the University of New York at Buffalo, the B.S. degree in physical therapy from New York University, and the B.S. and M.S. degrees in electrical engineering from Colorado State University.

His engineering areas of interest include signal/ image processing and neural networks. He is presently unemployed as an electrical engineer and is currently practicing physical therapy in Fort Col-

lins, $\mathrm{CO}$.

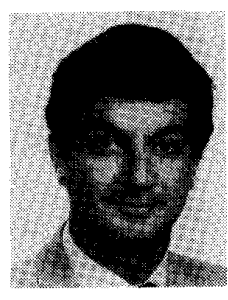

Mahmood R. Azimi-Sadjadi (M'81-SM'89) was born in Tehran, Iran, in 1952 . He received the B.S. degree from University of Tehran, Iran, in 1977, the M.S. and Ph.D. degrees from Imperial College, University of London, England, in 1978 and 1982, respectively, all in electrical engineering.

He served as an Assistant Professor in the Department of Electrical Computer Engineering, University of Michigan-Dearborn. Since 1986 he has been with the Department of Electrical Engineering, Colorado State University, where he is now an Associate Profes sor. His areas of interest are digital signal/image processing, multidimensional system theory and analysis, adaptive filtering, system identification, and neural networks. He is a co-author of the book, Digital Filtering in One and Two Dimensions (Plenum Press, 1989).

Dr. Azimi-Sadjadi is the recipient of the 1990 BATTELLE Summer Faculty Fellowship Award, and the 1984 DOW Chemical Outstanding Young Faculty Award of the American Society for Engineering Education. 